This item was submitted to Loughborough's Research Repository by the author.

Items in Figshare are protected by copyright, with all rights reserved, unless otherwise indicated.

\title{
Local fault location in meshed DC microgrids based on parameter estimation technique
}

\section{PLEASE CITE THE PUBLISHED VERSION}

https://doi.org/10.1109/JSYST.2021.3107905

\section{PUBLISHER}

Institute of Electrical and Electronics Engineers

\section{VERSION}

AM (Accepted Manuscript)

\section{PUBLISHER STATEMENT}

(c) 2021 IEEE. Personal use of this material is permitted. Permission from IEEE must be obtained for all other uses, in any current or future media, including reprinting/republishing this material for advertising or promotional purposes, creating new collective works, for resale or redistribution to servers or lists, or reuse of any copyrighted component of this work in other works.

\section{LICENCE}

\section{All Rights Reserved}

\section{REPOSITORY RECORD}

Bayati, Navid, Hamid Reza Baghaee, Amin Hajizadeh, Mohsen Soltani, Zhengyu Lin, and Mehdi Savaghebi. 2021. "Local Fault Location in Meshed DC Microgrids Based on Parameter Estimation Technique". Loughborough University. https://hdl.handle.net/2134/16438239.v1. 


\title{
Local Fault Location in Meshed DC Microgrids based on Parameter Estimation Technique
}

\author{
Navid Bayati, Hamid Reza Baghaee, Amin Hajizadeh, Senior Member, IEEE, Mohsen Soltani, Senior \\ Member, IEEE, Zhengyu Lin, Senior Member, IEEE, and Mehdi Savaghebi, Senior Member, IEEE
}

\begin{abstract}
Accurate locating of the faulty section is desired in DC microgrid due to the presence of power electronic converters and low-impedance cables. Some of the existing schemes consider power sources at only one end of the line; thus, assume that the fault current is injected from only one end of the line. This assumption is not true in the case of meshed DC microgrids, where fault current would be supplied from both ends. Moreover, existing communication-based methods require either a fast communication network or data synchronization. To address the aforementioned issues, this paper proposes a novel local fault location scheme for meshed DC microgrids. Low- and highimpedance faults are located by measuring the current by localized intelligent electronic device (LIED). Based on the parameter estimation approach, the fault location is estimated by sampling the peak values of the fault current. The effectiveness of the proposed strategy is evaluated based on offline digital timedomain simulations in MATLAB/Simulink software environment for a meshed test microgrid system and experimentally verified by implementing in a laboratory-scale hardware setup. Comparing the proposed method with other existing methods proves the effectiveness of the proposed technique for different types of faults.
\end{abstract}

Index Terms-fault location, DC microgrid, parameter estimation, protection.

\section{NOMENCLATURE}

$\mathrm{AC}$

$\mathrm{DC}$

PV

RER

LIED

SLG

C.B

$L$

$R$

C

$i_{C}$

$I_{L}$

Navid Bayati, and Mehdi Savaghebi are with the Electrical Engineering Section, Department of Mechanical and Electrical Engineering, University of Southern Denmark, Odense, Denmark (e-mail: navib@sdu.dk; mesa@sdu.dk). Amin Hajizadeh, and Mohsen Soltani are with the Department of Energy Technology, Aalborg University, Denmark (e-mails:aha@et.aau.dk; sms@et.aau.dk).

Hamid Reza Baghaee is with the Department of Electrical Engineering, Amirkabir University of Technology, Iran (e-mail: hrbaghaee@aut.ac.ir).

Zhengyu Lin is with the Wolfson School of Mechanical, Electrical and Manufacturing Engineering, Loughborough University, U.K (email: z.lin@,ieee.org)

$\begin{array}{ll}I_{0} & \begin{array}{l}\text { Initial value of the capacitor current } \\ V_{0}\end{array} \\ L_{1} & \begin{array}{l}\text { Initial value of the capacitor voltage } \\ \text { Line inductance between LIED and fault } \\ \text { location, }\end{array} \\ R_{1} & \begin{array}{l}\text { Line Resistance between LIED and fault } \\ \text { location, }\end{array} \\ R_{f} & \text { Fault resistance, } \\ i_{f}(t) & \text { Fault current, and } \\ P & \text { Coefficient of Taylor series, } \\ C_{\text {communication }} & \text { Cost of the communication link, } \\ C_{\text {equipment }} & \text { Cost of additional equipment, } \\ C_{\text {Sensors }} & \text { Cost of sensors } \\ C_{\text {IED }} & \text { Cost of IED } \\ C_{\text {workforce }} & \text { Cost of the required work force, }\end{array}$

\section{INTRODUCTION}

$\mathrm{D}$ C microgrids are an energy-efficient solution for the applications where the majority of electronic loads consuming DC power and DC sources like batteries, fuel cells, and PV array supply the system [1]. DC microgrids reduce the number of conversion stages compared to AC systems, and therefore reduce power losses [1].

Fault location schemes estimate the distance of fault, and the accuracy of these schemes is one of the key elements of fast maintenance and restoration of DC microgrids after faults. Fault location methods can be categorized into two groups, local and communication-based schemes. In communication-based fault location schemes, the measured data from both ends of lines are sent to the protection system through communication channels to locate the fault. However, the communication channel increases cost, noise, and failure probability.

Typically, in conventional centralized power systems, the fault current flow is unidirectional, and local current-based relays can detect or locate the fault [3]. However, in DC microgrids, the energy resources are connected in different locations, and each RER injects a share of fault current. Therefore, the fault current in these systems is bidirectional, and conventional localized techniques cannot be implemented in the DC microgrids [4].

\section{A. Related Works}

The protection of DC systems is widely studied in recent research works, and the suggested methods can be categorized as fault detection, [5]-[7], and fault location schemes [8]-[10]. 
In [5], the fault and faulty section is detected by using frequency oscillations, which are measured and compared using intelligence electric devices at both ends of each line segment. The principle of threshold violation is used in [6] to detect the fault in DC microgrids quickly. However, due to the concept of fault detection methods, they cannot locate the faulty position. To improve the reliability of protection systems, in [7], a backup protection system is suggested and uses a sequential analyzing technique. On the other hand, the fault location techniques in recent studies use an additional fault location module [8], the slope of fault current [9], and the Pearson correlation coefficient [10]. However, the existing works cannot accurately locate the high-impedance faults in meshed configuration DC systems without using communication links or additional equipment, which increases the cost of the protection system or reduces the reliability of the entire system.

Locating fault distance in DC microgrids is a crucial task for the restoration and maintenance of these systems, especially in critical systems such as islands, space stations, maritime microgrids, and applications with underground cables. Typically, the fault distance can be determined by offline schemes after the detection and isolation of the fault.

Fault location schemes can be categorized into two main groups, passive and active. Passive fault location schemes are based on measuring the data of currents or voltages. Active schemes are based on signal injection or deploying auxiliary equipment and analyzing the reflected signals [11]. Moreover, fault location methods can also be allocated into two groups of online and offline methods. In online methods, the fault location is estimated during the fault, and offline methods calculate the fault distance after clearing of fault [12].

Some of the previous works have proposed injecting the current into the cable using the external circuit and analyzing the behavior of the injected current to determine the location of fault [13]. In [14], a power probe unit has been proposed to inject the DC signals by a converter-based unit into the cable. This method operates well. However, using additional equipment increases the cost as well as human workload.

An online fault location estimation approach has been suggested in [15], which uses the traveling-wave-based technique for fault location. However, due to the low surge arrival time, this method is inaccurate if DC microgrids have small line lengths. A differential protection-based scheme for DC microgrids has been presented in [16], which demands reliable and fast communication links to send the data to the protection devices. However, the probability of data loss and communication failure and also the high cost of differential protection limit the application of these schemes. A localized technique has been presented in [17] to estimate the fault distance using the local current, current derivative, and voltage. However, in this technique, the DC line is assumed to be supplied from only one side. Thus, this is not applicable in the DC microgrids, where most lines are connected from two sides to the sources or power electronic loads. Power electronic converters usually have a DC bus capacitor, which can inject a high rising current to the faulty location during the first milliseconds of the fault.

\section{B. Contribution of Research}

To the best of the author's knowledge, the proposed scheme has the highest accuracy and lowest cost among the existing local fault location systems [8]-[10] for DC microgrids. The differences between DC and AC, meshed and radial microgrids, and with or without communication link in protection systems lead to the following challenges which are addressed in this work:

1) AC-related features such as frequency and phase characteristics are no longer available, and thus, the authors focus on new features that may be used for fault location in DC microgrids.

2) The communication-based schemes used in DC microgrids would require a network infrastructure, high sampling rate, and filtering equipment, which might not be feasible in a low-cost DC microgrid deployment. Detailed fault analysis is represented in Section II.

The existing fault location methods require both ends' data, and therefore, they suffer from noise and delay. Therefore, the existing researches [15]-[16] cannot locate faults by using only the measured values of one end of the line segment. Consequently, in this research, the available features in one end of the faulty line in DC microgrids are explored, and their relationship with the fault location is investigated. Through extensive simulations and experimental tests, the new features and their relationship with fault location are discovered. A novel fault location scheme is designed for meshed DC microgrids Based on information from the current sensor of one end of the faulty line. The approach is validated with experimental and simulation studies.

The main contributions of this paper include as follows:

1) Revealing the relationship between the peak features of the transient current signal and the fault distance in meshed DC microgrids. During a fault, current sensors should only detect the oscillation of the transient signal.

2) Designing a scheme for estimating the fault distance based on the peak features by using only local measured values. The performance of the technique is evaluated in different scenarios, and the error is analyzed. An experimental setup is also utilized to test the method.

The evaluation results manifest the significance of the novel scheme as there are few studies performed on the local fault location of meshed DC microgrids. The general comparison of the proposed method and existing methods are represented in Table I. As can be seen, most existing techniques suffer from several disadvantages such as high cost, requiring communication link, low accuracy, and only can be implemented in the radial system. The proposed scheme tried to solve the mentioned issues to provide an accurate and lowcost fault location technique.In this paper, a localized protection technique is proposed for meshed DC microgrids. In the proposed strategy, due to the correlation of fault distance and transient current, LIED, and the current signal of one place is utilized to estimate the fault location and resistance. This scheme does not use any communication links. Therefore, it is low-cost and avoids problems of communication-dependent strategies such as noise, time-delay, and the probability of 
TABLE I.

THE GENERAL COMPARISON OF THE METHODS IN LITERATURE AND THE PROPOSED METHOD

\begin{tabular}{|c|c|c|}
\hline Method & Advantages & Disadvantages \\
\hline$[8]$ & $\begin{array}{l}\text { Fast, accurate, local, and applicable in both radial and mesh DC } \\
\text { systems }\end{array}$ & $\begin{array}{l}\text { Requiring two additional components increasing the cost, and complexity of } \\
\text { finding the optimum values of each component. }\end{array}$ \\
\hline [10] & Fast, accurate, and applicable in both mesh and radial systems & Requiring high sampling rate, and analysing data in different windows. \\
\hline$[14]$ & $\begin{array}{l}\text { Without requiring communication link and external units, } \\
\text { therefore, it is a low-cost method }\end{array}$ & $\begin{array}{l}\text { Low accuracy, and fault resistance, and only can be implemented in radial } \\
\text { systems }\end{array}$ \\
\hline [15] & Applicable in mesh systems, and high accuracy & Requiring communication link, and high sampling rate \\
\hline$[16]$ & Fast, and accurate & $\begin{array}{l}\text { Requiring communication link, and high sampling rate, only can be } \\
\text { implemented in radial systems, and without considering fault resistances }\end{array}$ \\
\hline$[17]$ & Local and low-cost method & $\begin{array}{l}\text { Low accuracy, and fault resistance, only can be implemented in in radial } \\
\text { systems. }\end{array}$ \\
\hline $\begin{array}{l}\text { Proposed } \\
\text { scheme }\end{array}$ & $\begin{array}{l}\text { Local, accurate, considering fault resistances, low sampling } \\
\text { rate, and can be implemented in mesh DC Microgrids }\end{array}$ & Only applicable on DC systems, \\
\hline
\end{tabular}

communication failure. Finally, the effectiveness of the proposed fault location strategy is validated through offline digital time-domain simulations in MATLAB/Simulink environment for various locations and fault resistance values in a test DC microgrid system and experimentally verified by implementing a lab-scale testbed.

The rest of the paper is organized as follows: In section II, the fault analysis details for DC microgrids are discussed. Section III presents the parameter estimation techniques of the proposed localized fault location estimation method. Simulation and experimental test results are provided in section IV. Finally, the main conclusions are stated in section V.

\section{FAULT ANALYSIS IN DC MiCROGRIDS}

A fault in the DC microgrid may cause a destructive condition for the converters. The IGBTs are blocked for selfprotecting, and the reverse diodes are exposed to an overcurrent [18]. Therefore, analyzing the fault characteristics of DC microgrids is essential.

\section{A. Fault Characteristics in DC Microgrids}

After the occurrence of a short-circuit fault, the DC link capacitors of both ends of the line cause a high rising current and inject a current to the faulty location until it reaches its peak. After the occurrence of a fault, the discharge state of the capacitor is started, and the equivalent circuit of the line will be changed to an RLC circuit. The current waveform of this equivalent circuit, from experimental tests, is depicted in Fig.

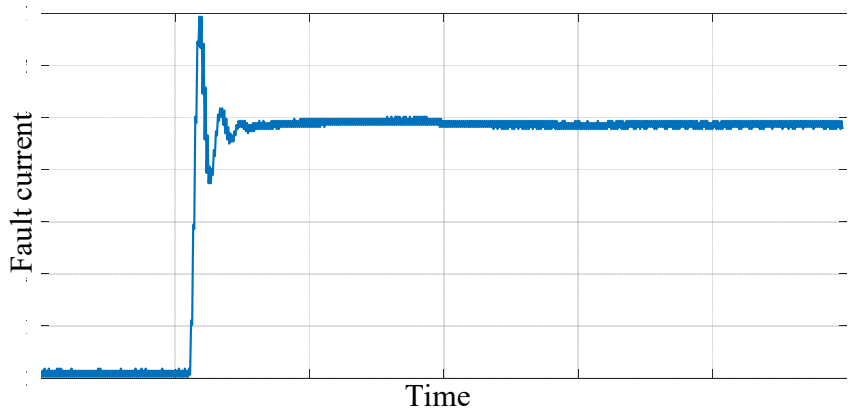

Fig. 1. The typical fault current in the DC system
1 , where the peak of this current could go around 10 times more than the nominal current of the cable. The cable and DC link capacitors of converters inject the transient current, and the RERs provide the steady-state current during the fault [19]. The DC-link capacitor current during fault can be obtained by:

$$
\frac{d^{2} i_{c}}{d t^{2}}+\frac{R}{L} \frac{d i_{c}}{d t}+\frac{1}{L C} i_{c}=0
$$

Moreover, after switching the converters during the fault, the energy of the inductance will be discharged into the cable. The current of this discharge is calculated by

$$
L \frac{d i_{L}}{d t}+i_{L} R=0
$$

Consequently, as shown in Fig. 1, the transient part of the fault current is made by $i_{L}$ and $i_{C}$. On the other hand, during the zero fault resistance faults, when the voltage at the fault location drops to zero, the capacitors of the lines are discharged entirely, and based on the state of (1), the fault current will be over or underdamped. The voltage of the capacitor of one end can be obtained by:

$$
V_{c}=\frac{1}{C} \int i_{c}(t) d t
$$

Therefore, by subtracting (1) and (3), the voltage of the capacitor is determined by:

$$
V_{c}(t)=\frac{e^{-\alpha t}}{\omega}\left(V_{0} \omega_{0} \sin (\omega t+\beta)-\frac{I_{0}}{C} \sin (\omega t)\right)
$$

Where,

$$
\left\{\begin{array}{c}
\alpha=\frac{R}{2 L} \\
\omega=\sqrt{\frac{1}{L C}-\frac{R^{2}}{4 L^{2}}} \\
\omega_{0}=\sqrt{\alpha^{2}+\omega^{2}} \\
\beta=\tan ^{-1}\left(\frac{\omega}{\alpha}\right)
\end{array}\right.
$$

Then, the time for full discharge of the capacitors, or the end of capacitor discharge state, is calculated by: 


$$
t=\frac{1}{\omega} \cot ^{-1}\left(\frac{I_{0}}{V_{0} \alpha C}+\frac{\alpha}{\omega}\right)
$$

Due to the low value of $C$ and $\omega$ in the DC microgrids, the time before voltage collapse in the DC systems is much less than the AC systems. Moreover, the fault current transient is divided into three different states: slow, medium, and fast fronttransients. Storage units, voltage-dependent loads, and control of converters can cause slow front-transient. The capacitors make the medium front-transient of filters, and by opening the switchgear, a transient recovery voltage makes a fast front transient [20]-[22].

\section{B. High-Impedance Fault Characteristics in DC Microgrids}

When a conductor approaches close to ground or another conductor through a high impedance, a high-impedance fault occurs. In these conditions, the fault current will have a lower magnitude compared to low-impedance faults, therefore, locating this type of faults is a challenging task. The highimpedance faults are very complex phenomena and presents a highly nonlinear performance. The characteristic of a high impedance faults is divided into three different stages, namely, buildup, shoulder, and nonlinearity. The model of high impedance faults in DC systems has been considered rarely. The model in [1] is utilized in this paper to accurately analyze the performance of high impedance faults, as follows

$$
\begin{aligned}
& \left\{\begin{array}{l}
i_{j+1}=i_{j}-\frac{R i_{j}+k / i_{j}^{1.2}+35-V_{D C} \sin \omega t}{R-1.2 k / i_{j}^{2.2}} \\
2 n \pi+\pi / 3<\omega t<2 n \pi+2 \pi / 3 \quad n=0,1,2, \ldots
\end{array}\right. \\
& R=\frac{1.2 k i_{j+1} / i_{j}^{2.2}-1.2 k / i_{j}^{2.2}-k / i_{j}^{1.2}-35+V_{D C} \sin \omega t}{i_{j+1}}
\end{aligned}
$$

Equation (7) is used in laboratory observations [1], and the constant value of $k$ can be calculated by experimental tests for different situations.

\section{Proposed Fault Location Method}

The proposed protection system consists of an LIED and a current sensor which are installed at each line to calculate the fault location. Conventional methods use the voltage and current data from both ends of the faulty line, and communication links are needed. In the proposed method, only the current from one side of the line is measured. Therefore, the cost of the system is less, and the concerns for the time-delay, noise, and probability of communication failure will be eliminated.

During the fault, different fault currents flow through each end of the lines. Therefore, based on the characteristics of the installed C.Bs, they will isolate the line by different operation times. Then, the equivalent circuit will be an RLC circuit. Therefore, at this stage, the proposed LIED circuit starts to sample fault current and locate the fault. On the other hand, two types of faults can occur in the DC lines, i.e. internal and external faults. Using the direction of fault current, the internal and external faults can be distinguished, and the proposed LIED only operates during the internal faults. Therefore, based on Fig. 2, the current $I$, which injects into the line segment, is indicated by positive and negative magnitudes during the internal and external faults, respectively.

In the proposed fault location method is shown in Fig. 2. After isolating the fault by C.Bs, the operation of the faulty segment will change to the RLC circuit. Therefore, the LIED starts to measure the current by a sampling rate of $\mu$. Although a higher sampling rate will provide more current samples, it causes more cost on the selection of sensors. Based on [5], in DC systems, the accuracy of fault location methods for sampling rates from $25 \mathrm{kHz}$ to $100 \mathrm{kHz}$ is almost constant. Therefore, in this paper, the sampling rate of $25 \mathrm{kHz}$ is selected for sensors. During this stage, the equivalent circuit of the system is depicted in Fig. 3.

Once the C.Bs isolated the line from both sides, due to the opening of the C.B $B_{2}$ and C. $B_{1}$, an RLC loop is formed, and the current of this loop is calculated as follows:

$$
\frac{d^{2} i_{f}(t)}{d t^{2}}+\frac{R_{1}+R_{f}}{L_{1}} \frac{d i_{f}(t)}{d t}+\frac{1}{L_{1} C} i_{f}(t)=0
$$

Thus, the value of $i_{f}$ as a function of time can be written as

$$
i_{f}(t)=e^{-\alpha t}(D \cos (\omega t)+E \sin (\omega t))
$$

where the values of $\alpha$ and $\omega$ can be obtained by:

$$
\left\{\begin{array}{c}
\alpha=\frac{R_{1}+R_{f}}{2 L_{1}} \\
\omega=0.5 \sqrt{\frac{4}{L_{1} C}-\frac{\left(R_{1}+R_{f}\right)^{2}}{L_{1}^{2}}}
\end{array}\right.
$$

The current during a fault can be over, under, and critically damped based on the characteristic of the system. In DC microgrids, the DC link capacitor has a low value. Therefore, the condition of underdamped of fault current will be satisfied if:

$$
\alpha^{2} \leq \omega^{2}
$$

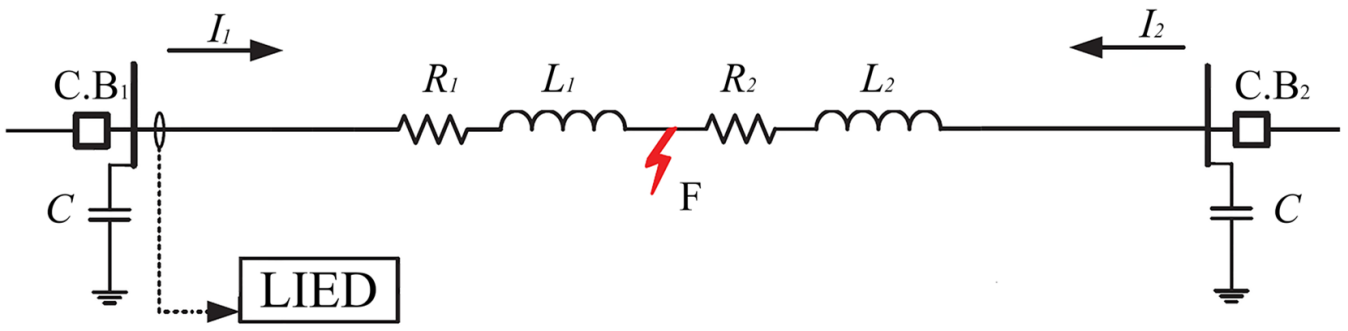

Fig. 2. Internal fault case in the DC microgrid. 


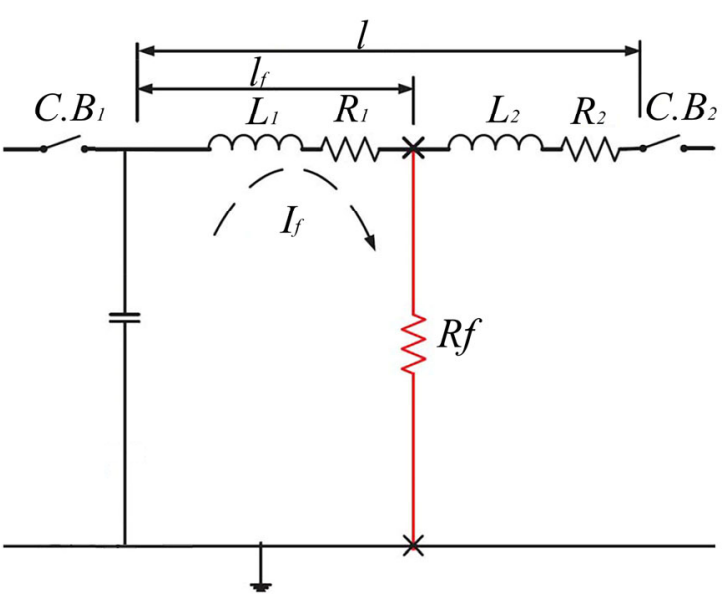

Fig.3. Structure of faulty line segment after isolation

Then,

$$
\frac{\left(R_{1}+R_{f}\right)^{2}}{L_{1}} \leq \frac{2}{C}
$$

Hence, due to the extremely low value of DC-link capacitor of low-voltage DC microgrids, (12) is always satisfied, even for high fault resistances. Note that based on the system characteristics in Table II, the condition of (12) is satisfied.

Due to the four unknown parameters in (9), an estimation method should be used to generate more equations to find the unknown parameters. Therefore, by using the Taylor series, (9) can be rewritten with neglecting higher-order terms as

$$
i_{f}(t)=P_{1} t^{3}+P_{2} t^{2}+P_{3} t+P_{4}
$$

where,

$$
\left\{\begin{array}{c}
P_{1}=\frac{D}{6}\left(2 \alpha \omega^{2}-\alpha^{3}-\alpha^{2} \omega\right)+\frac{E}{6}\left(3 \alpha^{2} \omega-\omega^{3}\right) \\
P_{2}=\frac{D}{2}\left(\alpha^{2}-\omega^{2}\right)-E \alpha \omega \\
P_{3}=-D \alpha+\omega E \\
P_{4}=D
\end{array}\right.
$$

Therefore, the LIED requires measuring the current with only four samples to estimates the values of $P_{1}, P_{2}, P_{3}$, and $P_{4}$ to fit the underdamped current of fault (Fig. 4 ) to a cubic equation.
By solving (14), $D, E, \alpha$, and $\omega$ are calculated. Consequently, by substitution of $D, E$, $\alpha$, and $\omega$ in (10), the values of $R_{l}, R_{f}$, and $L_{l}$ will be obtained. It could be noted that for calculating $R_{l}$, and $L_{1}$, they should be replaced with $R_{s} d$ and $L_{s} d$, in which $R_{s}$ and $L_{s}$ are the resistance and inductance of each meter of line, respectively, and $d$ is the fault distance. It should be noted that the peak values are identified by comparing samples to previous and next samples. If the previous samples are increasing continuously, and the next samples are decreasing continuously, it is identified as one of the peak points of the fault current.

\section{Simulation AND EXPERIMENTAL RESUltS}

Offline digital time-domain simulation studies are performed in MATLAB/Simulink, including different fault distances and resistances in a $3-\mathrm{km}$ line segment of a DC microgrid, as shown in Fig. 2. The detailed parameters of this segment are represented in Table II. Also, to prove the effectiveness of the proposed scheme in the fault location, it is tested experimentally. In the laboratory test, each $1 \mathrm{~km}$ line segment is represented by a high-resistance inductance. Furthermore, in this paper, IEEE Std C37.114 has been used for the selection of the error and accuracy of the proposed method. Moreover, this standard has been used during the test to implement the data and required equipment.

\section{A. Offline Digital Time-Domain Simulation}

As shown in Fig. 2, an SLG fault with different values of fault resistance is created. A line to ground fault with fault resistance of $0.2 \Omega$ is created at time $t=0.05 \mathrm{~s}$ in the middle of the line segment. Current and voltage through the LIED are shown in Fig. 5 (a) and Fig. 5 (b), respectively. Also, the values of samples of the current are shown in Fig. 5 (a). For the same fault, the values of the peak of fault current are shown in Fig. 6. According to this figure, by increasing the fault resistance, the peak magnitudes will reduce. However, due to the underdamped behavior of fault current, the required samples of the proposed method will be the same for different fault

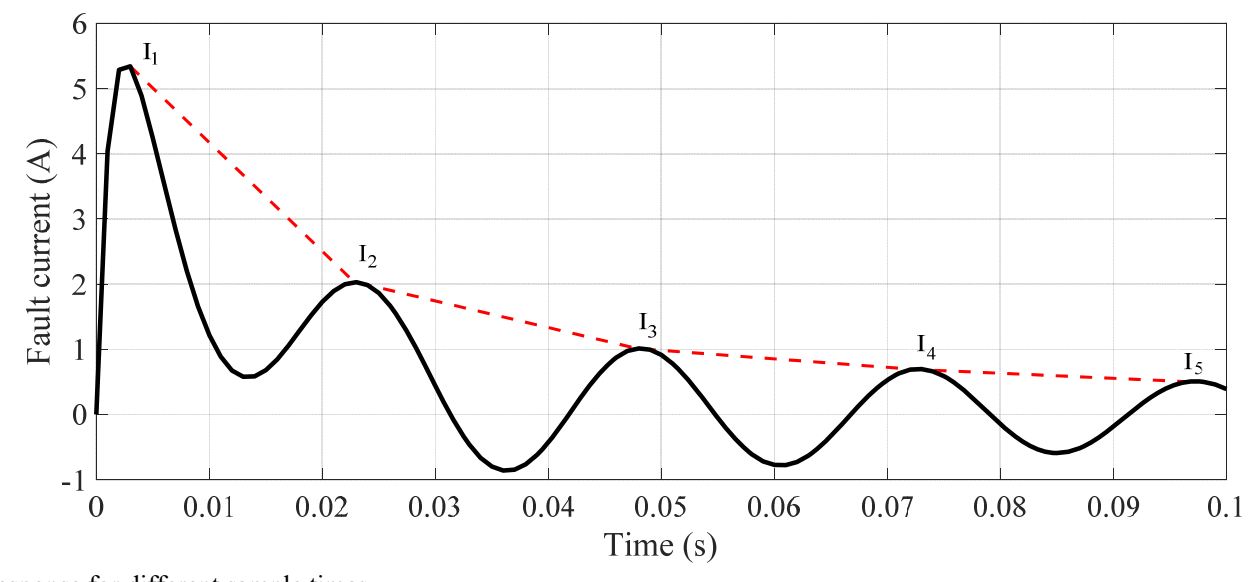

Fig. 4. Probe current response for different sample times 
resistances.

At the second stage of the protection method, the location of the fault is estimated by using a LIED without using any communication links and only by using the data of the LIED. By applying the proposed technique, the distance of the fault from the LIED place is estimated and shown in Fig. 7 for fault resistance between 1 and $6 \Omega$. The proposed method is applied for the faults in two different locations with different fault resistances, and the results show that the value of error for faults which are $2 \mathrm{~km}$ far from the LIED is less than $6 \%$ and for fault with the distance of $750 \mathrm{~m}$, this error is maximum $4 \%$. It is important to note that based on the standards, the currents higher than $125 \%$ of normal current should be detected as fault current. Thus, the maximum value of fault resistance in this system will be $6 \Omega$. The results of the estimated fault distances are shown in Table III, and it demonstrates that increasing the fault resistance increases the value of fault location estimation error. The fault location methods are a function of $R / R_{f}$, and decreasing this value increases the fault location error. However, it is still in an acceptable range.

On the other hand, as can be seen in (8)-(12), the impact of line resistance, $R_{l}$, and fault resistance, $R_{f}$, on the performance of the proposed method is the same. Moreover, by determining the value of error as the difference of estimated and actual fault

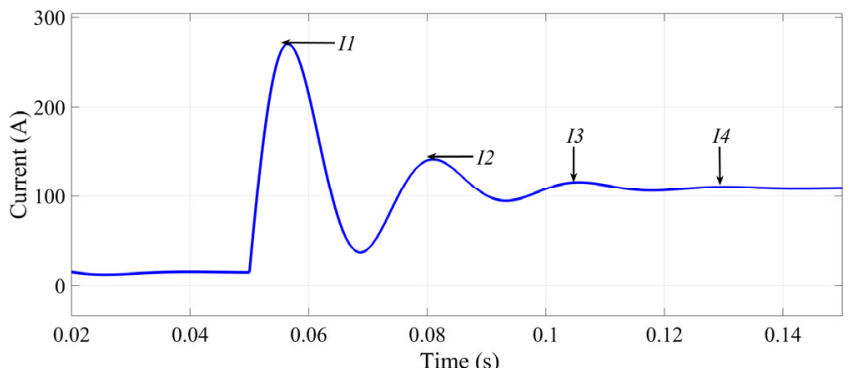

(a)

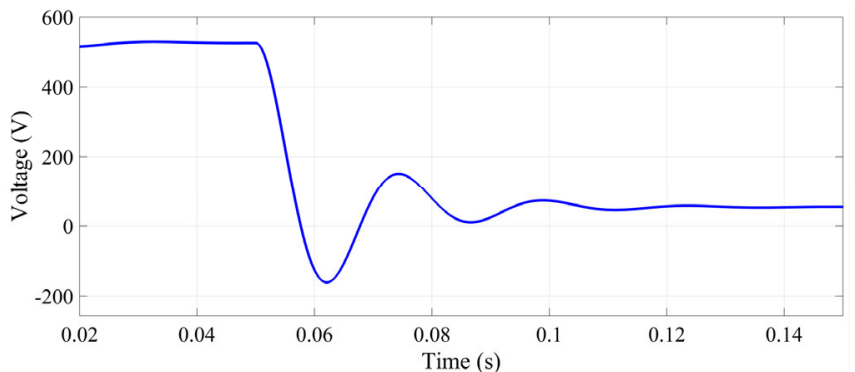

(b)

Fig. 5. (a) fault current waveform at LIED place (b) fault current waveform at LIED place

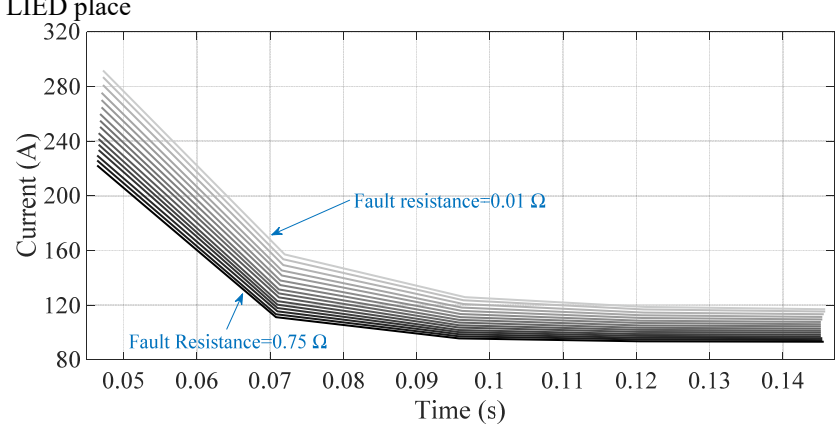

Fig. 6. The values of peak of current for different fault resistances currents, respectively (13) and (9), a sensitivity analysis is performed, and the sensitivity of error function to $R_{f}$ is determined by:

$$
S_{R_{f}}^{\text {Error }}=\frac{R_{f}}{\text { Error }} \times \frac{\partial \text { Error }}{\partial R_{f}}
$$

in which, the error of fault location method has a higher value during higher values of $R_{f}$. Consequently, it shows that the LIED has a higher error, during the high fault resistances or faults with a high distance, compared to low impedance faults, as also shown in Table III.

\section{B. Experimental Validation}

The proposed fault location estimation technique is validated on a lab-scale hardware setup. Two power supplies, EA-PS 9360 and APM-SP800VDC, are used in the experiment. The constant current power supply, EA-PS 9360, is connected by a $\mathrm{DC} / \mathrm{DC}$ converter as a power source, and the DC bus is implemented by the constant voltage power supply APM-

TABLE II.

SIMULATION PARAMETERS

\begin{tabular}{ll}
\hline \hline Component & Rating \\
\hline Line length & $3 \mathrm{~km}$ \\
Inductance per km & $10 \mathrm{mH}$ \\
Resistance per km & $160 \mathrm{~m} \Omega$ \\
DC link capacitor & $0.25 \mathrm{mF}$ \\
Nominal Voltage & $480 \mathrm{~V}$ \\
Maximum fault resistance & $6 \Omega$
\end{tabular}

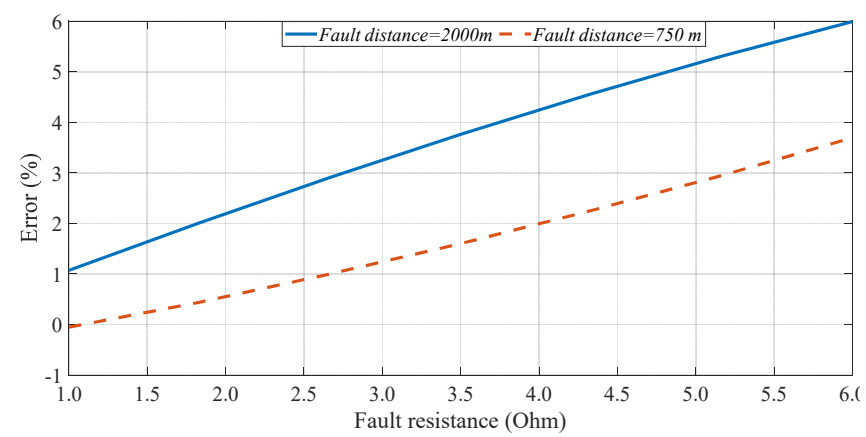

Fig. 7. Fault location estimation by using the proposed localized method

TABLE III.

THE RESULTS OF FAULT LOCATION ESTIMATION FOR SIMULATION TESTS

\begin{tabular}{|c|c|c|c|}
\hline $\begin{array}{l}\text { Fault resistance } \\
(\Omega)\end{array}$ & $\begin{array}{ll}\text { Actual fault } \\
\text { location (m) }\end{array}$ & $\begin{array}{ll}\text { Estimated } & \text { fault } \\
\text { location }(\mathrm{m}) & \end{array}$ & $\begin{array}{l}\text { Error } \\
(\%)\end{array}$ \\
\hline 1.0 & 500 & 499.55 & 0.09 \\
\hline 1.2 & 500 & 499.45 & 0.11 \\
\hline 1.4 & 750 & 748.72 & 0.17 \\
\hline 1.9 & 750 & 746.41 & 0.48 \\
\hline 3.4 & 1000 & 985.79 & 1.42 \\
\hline 4.5 & 1000 & 969.02 & 3.10 \\
\hline 5.3 & 2000 & 1896 & 5.20 \\
\hline 6.0 & 2000 & 1880 & 6.00 \\
\hline
\end{tabular}


SP800VDC. The experimental setup diagram is depicted in Fig. 8, and the photos of the practical setup are shown in Fig. 9 (a)(c). The system has a nominal DC bus voltage of $24 \mathrm{~V} \mathrm{DC}$, and each inductor is equivalent to a $1-\mathrm{km}$ cable, by the resistance of $0.16 \Omega$ and inductance of $0.01 \mathrm{mH}$. A $24 \mathrm{~V} \mathrm{DC}$ motor is also connected to the DC power supply as an electrical load. A dSPACE controller is used to record the experimental data and investigate the performance of the proposed scheme.

Fig. 10 (a) and (b) show the experimental results of a high resistance fault with fault resistance of 2 and $3 \Omega$ at $2 \mathrm{~km}$, and $1 \mathrm{~km}$ away from the LIED, respectively. The magnitude of fault current is $5.84 \mathrm{~A}$ for fault at $2 \mathrm{~km}$, and $4.81 \mathrm{~A}$ for fault at $1 \mathrm{~km}$, and they will be damped to the steady-state fault current after approximately $200 \mathrm{~ms}$.

Moreover, the experimental result of the fault current for a fault at $2 \mathrm{~km}$ distance from LIED with fault resistance $1.4 \Omega$ is shown in Fig. 11. The maximum values of fault current are calculated and shown in Fig. 11. The values of $I_{1}, I_{2}, I_{3}$, and $I_{4}$ are inserted to (14) in dSPACE environment for calculating the fault location.

By applying the proposed fault location estimation method to the experimental results, the location of the fault is estimated

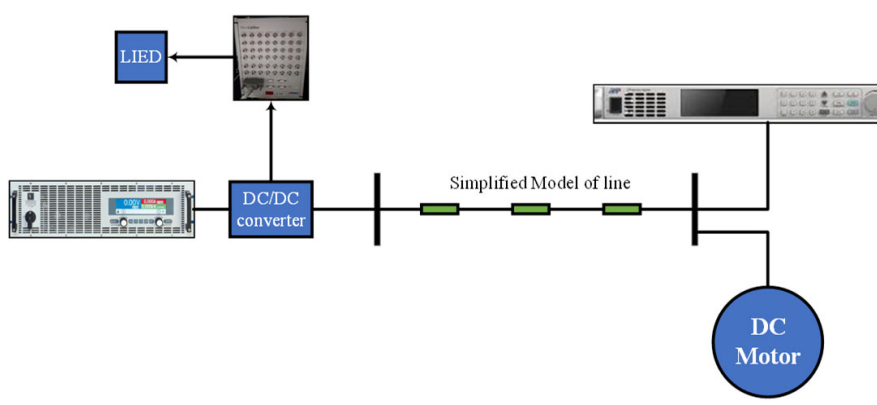

Fig. 8. The diagram of the experimental setup

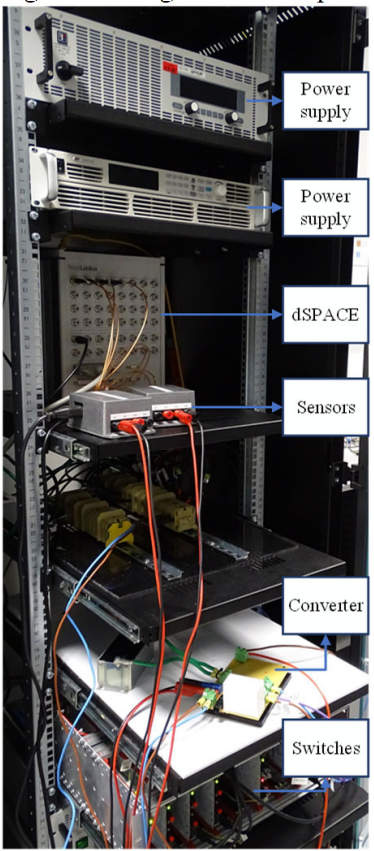

(a)

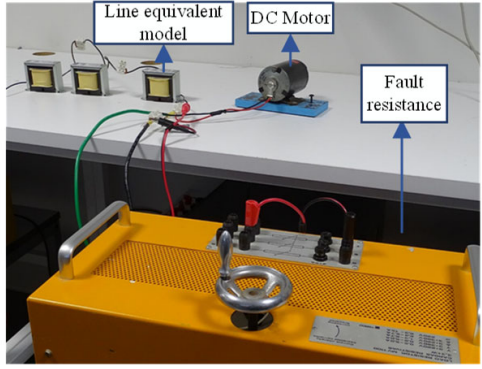

(b)

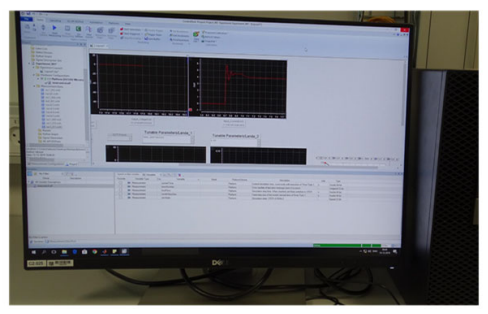

(c)
Fig. 9. The experimental setup for evaluating the proposed method (a) DC bus components (b) simplified model of line and load (c) Dspace user interface for different fault distances and resistances. These values are presented in Table IV. As expected, by increasing the values of fault distance and resistance, the value of error increases. However, the results indicate that the maximum value of error is $2.23 \%$, proving the effectiveness of the proposed technique. Therefore, these results explain that the line segment is protected. The location of the fault is estimated by using localized protection, which reduces the cost, failure probability of communication line, delay, and noises. This scheme has potential advantages for practical applications as it enables the use of localized data for DC microgrid protection and improves the sensitivity and reliability of the protection system.

The evaluation of the proposed method for different fault resistances is presented in Fig. 12. The experimental tests are performed for fault resistances up to $6 \Omega$; therefore, to investigate the performance of the proposed method for fault resistance up to $10 \Omega$, a curve fitting analysis was added to the collected data. As shown in Fig. 12, for high-impedance faults, the value of error is increased to $6.4 \%$, which is in an acceptable

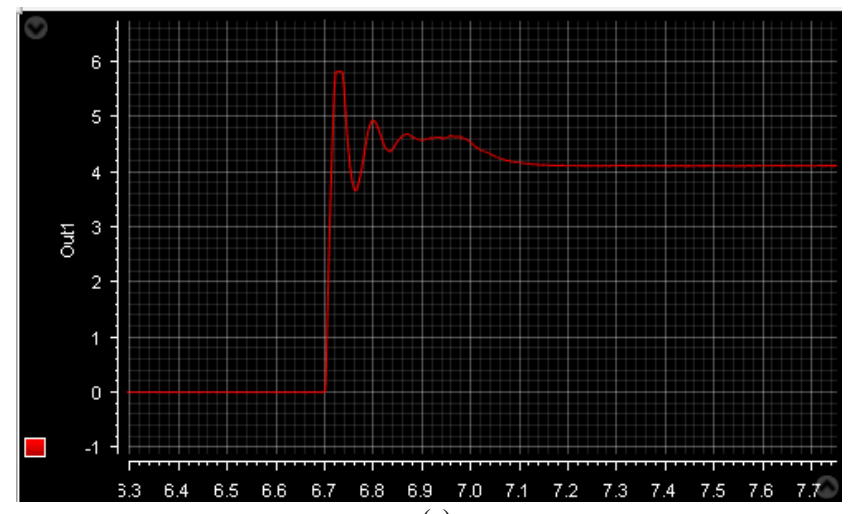

(a)

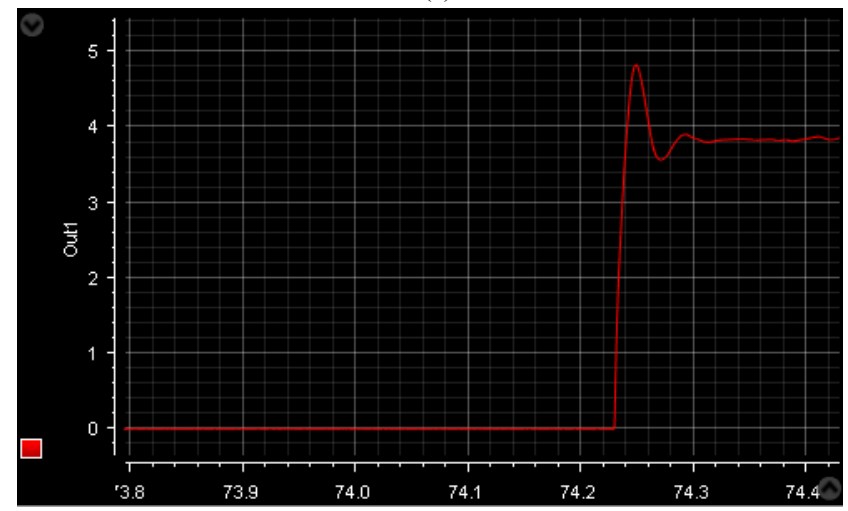

(b)

Fig. 10. The fault current of the LIED at one end of line for fault resistance of (a) $2 \Omega \mathrm{s}$ with $2 \mathrm{~km}$ (b) $3 \Omega \mathrm{s}$ with $1 \mathrm{~km}$ distance from LIED

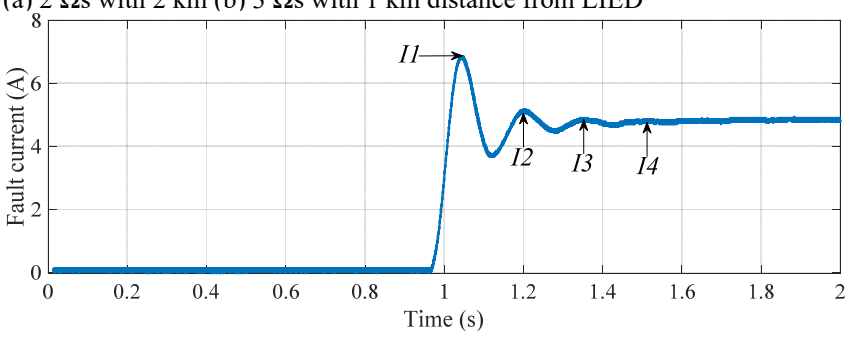

Fig. 11. The values of the fault current peaks for fault resistance $1.4 \Omega$ at fault distance $2 \mathrm{~km}$ 


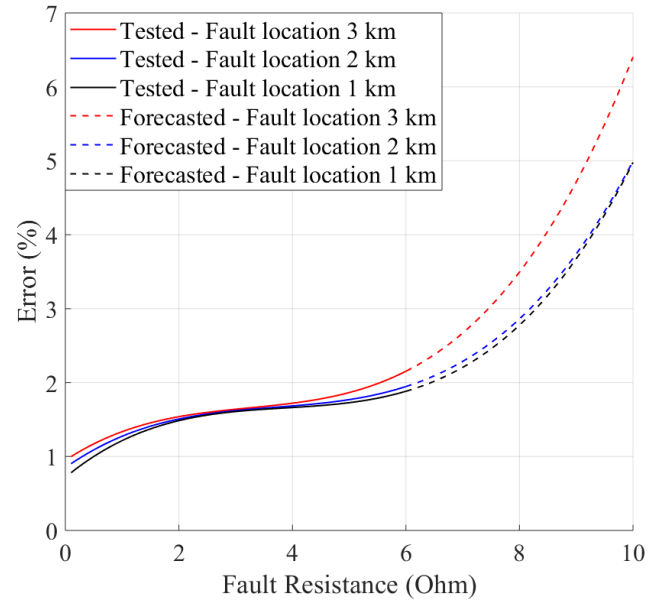

Fig. 12. Expected fault location error for different fault resistances and locations

TABLE IV.

\begin{tabular}{llll}
\multicolumn{4}{l}{ THE RESULTS OF FAULT LOCATION ESTIMATION FOR EXPERIMENTAL TESTS } \\
\hline $\begin{array}{l}\text { Fault } \\
\text { resistance }(\Omega)\end{array}$ & $\begin{array}{l}\text { Actual } \\
\text { location }(\mathrm{m})\end{array}$ & $\begin{array}{l}\text { fault } \\
(\mathrm{m})\end{array}$ & $\begin{array}{l}\text { Estimated fault location } \\
(\%)\end{array}$ \\
\hline 1.4 & 1000 & 986 & 1.36 \\
2.2 & 1000 & 985 & 1.48 \\
3.2 & 1000 & 983 & 1.66 \\
3.0 & 2000 & 1967 & 1.64 \\
4.8 & 2000 & 1965 & 1.74 \\
5.3 & 2000 & 1964 & 1.79 \\
5.5 & 3000 & 2939 & 2.03 \\
6.0 & 3000 & 2936 & 2.11 \\
6.2 & 3000 & 2933 & 2.23 \\
\hline
\end{tabular}

range. Therefore, the accuracy of the proposed method for sorely high fault resistance is demonstrated.

\section{Comparison with Other Reported Methods}

The results of the proposed technique are compared with other previously-reported researches published in [8], [9], [14], [17], and [23]-[28], as shown in Table V. Due to using two protection devices at each line segment in [8], and [23]-[27], the cost of these methods is higher than the proposed local scheme. Also, the communication-based methods in [24]-[26] and [28] are affected by noise and communication delay. Another important factor for evaluating a fault location method is the values of maximum fault resistance and error. The lowest value of error is for [20]; however, in this method, only short lines are investigated. The error of the reported method in [23] is around $0.4 \%$ for faults up to $2 \Omega$, but this method requires a voltageclamping diode, and high sampling rate current and voltage sensors at both ends of every line segment. The maximum values of considered fault resistance in existing methods are shown in Table V. Furthermore, the application of some of the compared researches is limited to only radial DC systems, such as [9], [14], [17], and [24]. In contrast, the proposed method is applicable to both radial and mesh DC systems. The maximum considered fault resistance for the proposed method is $6 \Omega$, and this scheme has an acceptable error of $6 \%$, which is lower than other fault location methods.

In Fig. 13, the comparative assessments of existing works with the proposed scheme are shown. The qualitative performance evaluation of each scheme based on the performance parameters of Table $\mathrm{V}$ is represented as a diagram, where parameters are qualitatively evaluated for providing fair taxation on different approaches. The evaluation is presented in

TABLE V.

THE QUALITATIVE AND QUANTITATIVE OF COMPARISON OF THE PROPOSED METHOD WITH OTHER METHODS

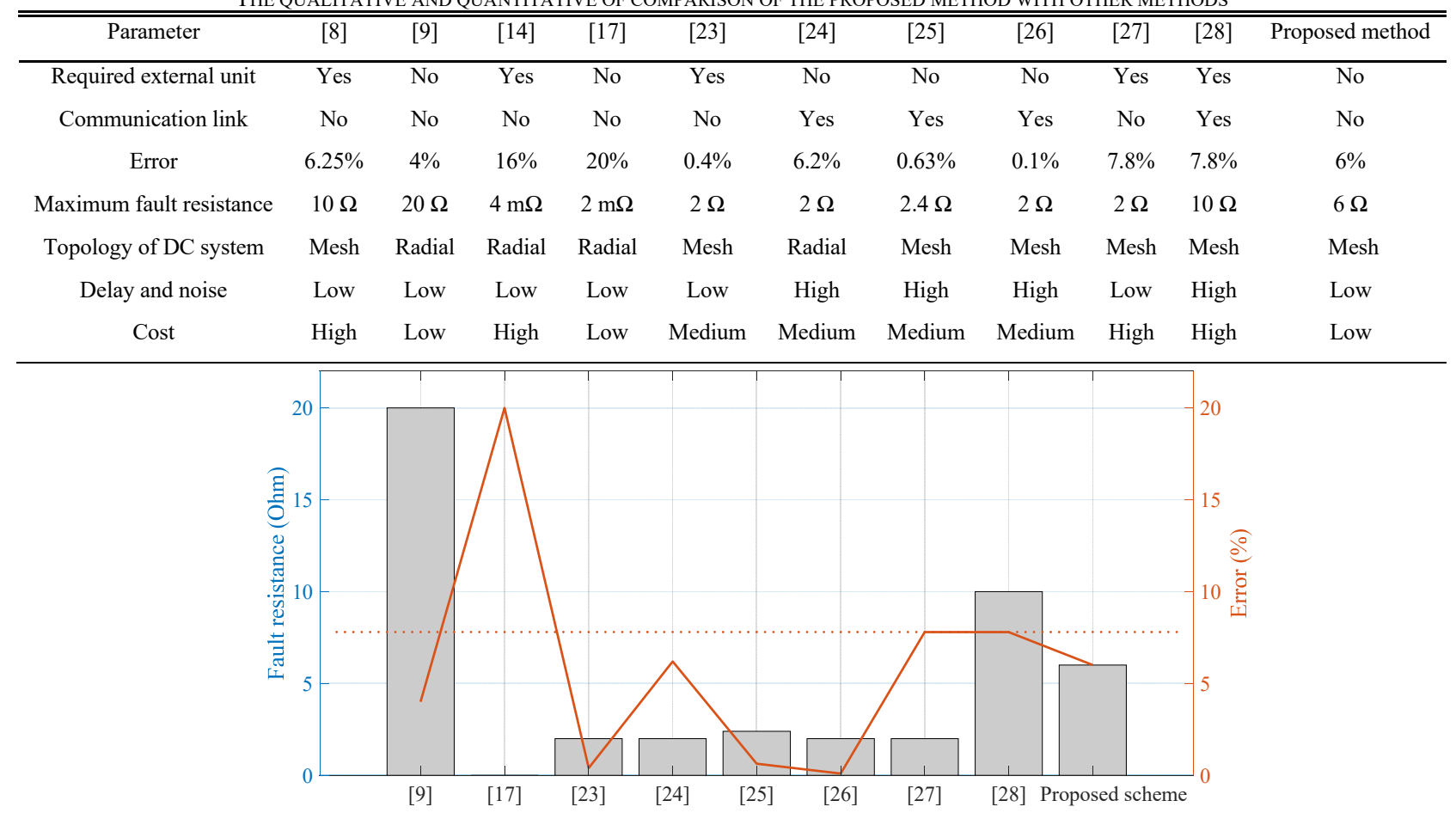

Fig. 13. The maximum error and fault resistance of existing methods and proposed method 
two diagrams depicted in Fig. 13. In this figure, the minimum error values of fault location estimation for existing local methods are depicted. The maximum error of the proposed method is lower than the minimum errors of other local-based methods, except [9], which only can be implemented in radial systems. The error of communication-based fault location methods is lower than the proposed method, however, they can only locate low-impedance faults, and the cost of implementation is high.

The comparative results prove that the proposed technique has lower error and higher accuracy than the other local schemes and can estimate the location of the high fault resistances. As can be seen from the results, by implementing the proposed protection method and corresponding protection equipment, it can be concluded that the proposed LIED scheme is applicable, and the efficiency, accuracy, and feasibility of the scheme are shown compared to the different existed methods. In the experimental and simulation section, the maximum fault resistance is considered as a high value, which causes a low fault current. As a result, faults are located in different locations with an error value of less than $6 \%$, which seems acceptable.

The cost of protection systems can be evaluated by the following equation:

$C=C_{\text {communication }}+C_{\text {equipment }}+C_{\text {Sensors }}+C_{I E D}+C_{\text {workforce }}$

The overall cost of a protection system comprises the cost of the communication equipment (physical link and interfaces), the installation cost (work and labor), the sensors, and its IEDs. The cost comparison analysis of existing protection systems has shown that localized protection systems offer practically the lowest cost option, which is due to the unnecessity of communication links, low installation cost, low cost of labor (no specialized expertise), and affordable sensors. For more detailed cost evaluations of [8], [9], [14], [17], [23]-[28], and the proposed work, the detailed required equipment of all methods is shown in Table VI.

Consequently, due to the two protection devices for each line segments, installation workloads, additional equipment, and communication link, reported methods in [8], [14], [27], and
[28] are categorized as high cost methods. The suggested methods in [9], and [17] are categorized as low-cost methods, due to the lack of additional requirements. Although a detailed cost analysis has been carried out in this work, the cost of the proposed scheme seems reasonable. The proposed method only requires the current sensors at one side of each line segment without any additional requirements and communication links, which can categorize the proposed scheme among low-cost schemes.

\section{Discussion}

The proposed protection strategy is clearly able to locate the fault in DC microgrids in different locations with different values of fault resistances. This is especially validated and investigated in a double-side-fed line segments, as shown in Fig. 2 and 8, which also can be applicable in loop configuration systems. Because most lines in a DC microgrid have a bidirectional power flow, implementing a protection method for such line segments is essential. Moreover, placing the proposed LIED in each line is more economical than installing relays at both ends of each line. Due to the local performance of the proposed method, the cost, communication failure, and noise are reduced. Consequently, the reliability of the proposed scheme will be higher than other communication-based protection methods. However, due to the existing noise in measurement devices, the fault current of the experimental tests includes measurement noises, but the error of it does not exceed $6 \%$. Furthermore, the effectiveness of the proposed method is investigated during high-impedance faults, which cause a lowmagnitude fault current. During these cases, as can be seen in Table IV, by changing the values of fault resistances and locations, the values of error are varied to a maximum of $2.23 \%$. As expected, increasing the value of fault resistance and location increases the value of error. However, this value is still in an acceptable range.

In case of variation of fault current from RER side, due to the consideration of reduction of fault current by fault resistance in the proposed scheme, the proposed method is immune against variation of fault current by RERs. For example, as shown in

TABLE VI.

THE DETAILED COST COMPARISON OF EXISTING AND PROPOSED SCHEMES

\begin{tabular}{|c|c|c|c|c|c|c|c|c|c|c|c|}
\hline Parameter & [8] & [9] & [14] & {$[17][17]$} & [23] & [24] & {$[25]$} & [26] & [27] & [28] & $\begin{array}{l}\text { Proposed } \\
\text { method }\end{array}$ \\
\hline $\begin{array}{c}\text { Communication } \\
\text { link }\end{array}$ & No & No & No & No & No & Yes & Yes & Yes & No & Yes & No \\
\hline $\begin{array}{l}\text { Additional } \\
\text { protection } \\
\text { system } \\
\text { equipment }\end{array}$ & $\begin{array}{c}\text { Two } \\
\text { inductances, } \\
\text { thyristors, } \\
\text { switches }\end{array}$ & -- & $\begin{array}{l}\text { Current } \\
\text { injector }\end{array}$ & -- & $\begin{array}{l}\text { Voltage- } \\
\text { clamping } \\
\text { diode }\end{array}$ & -- & -- & -- & $\begin{array}{l}\text { Resistor, } \\
\text { inductor, } \\
\text { capacitor, } \\
\text { switch }\end{array}$ & $\begin{array}{c}\text { Two voltage } \\
\text { divider } \\
\text { measurement }\end{array}$ & -- \\
\hline $\begin{array}{l}\text { Sampling rate } \\
\text { of sensors }\end{array}$ & $20 \mathrm{kHz}$ & $\begin{array}{c}1.3 \\
\mathrm{kHz}\end{array}$ & $1 \mathrm{kHz}$ & $20 \mathrm{kHz}$ & $100 \mathrm{kHz}$ & $5 \mathrm{kHz}$ & $80 \mathrm{kHz}$ & $2.2 \mathrm{kHz}$ & $2 \mathrm{kHz}$ & $4 \mathrm{kHz}$ & $25 \mathrm{kHz}$ \\
\hline Type of sensor & Current & Current & $\begin{array}{l}\text { Voltage } \\
\text { and } \\
\text { current }\end{array}$ & $\begin{array}{l}\text { Voltage } \\
\text { and } \\
\text { current }\end{array}$ & $\begin{array}{l}\text { Voltage } \\
\text { and } \\
\text { current }\end{array}$ & $\begin{array}{l}\text { Voltage } \\
\text { and } \\
\text { current }\end{array}$ & Current & $\begin{array}{l}\text { Voltage } \\
\text { and } \\
\text { current }\end{array}$ & Current & $\begin{array}{c}\text { Voltage and } \\
\text { current }\end{array}$ & Current \\
\hline $\begin{array}{l}\text { Rquire } \\
\text { protection units } \\
\text { for each line } \\
\text { segment }\end{array}$ & Two & One & One & One & Two & Two & Two & Two & Two & One & One \\
\hline Cost & High & Low & High & Low & Medium & Medium & Medium & Medium & High & High & Low \\
\hline
\end{tabular}


Fig. 6, the value of the peak of fault current is reduced from 280 A to $220 \mathrm{~A}$. Moreover, in the case of differentiating the faults and RER current injection, as can be seen in Fig. 2, if the RER injects fault current by $I_{2}$, it cannot impact $I_{1}$, and therefore, LIED will be immune against it. On the other hand, if the RER injects fault current by $I_{l}$, it will impact the behavior of measured fault current, for example, as depicted in Fig. 5, and Fig. 10, in which this fault current is analyzed for the location of the fault. Consequently, the proposed method is immune to the variation in performance of the RER.

\section{Conclusion}

The previous DC microgrid protection methods are effective theoretically, but almost all of them need communication links or cannot be effective during high fault resistances. In this paper, a fault location estimation technique was proposed to locate the fault distance by using local measured values. Thus, the cost, failure probability due to the communication line failure, and time-delay are minimized. The proposed technique uses a cubic equation estimation of seen resistance at each LIED during the fault. The obtained results proved the accuracy of the proposed technique for different fault distances and resistances. The proposed scheme is examined through extensive simulations and lab experiments considering high fault resistances to prove its effectiveness and accuracy. Moreover, the proposed protection strategy was compared with other reported techniques, and the comparative results indicated that the proposed method has lower error and higher accuracy compared to the other local schemes and can accurately estimate the location of the high-impedance faults.

\section{ACKNOWLEDGMENT}

Authors would like to thank Energy Cluster Denmark, and Danfoss Drives A/S for their support to the research work.

\section{REFERENCES}

[1] Bayati N, Baghaee HR, Hajizadeh A, Soltani M, Lin Z. Mathematical morphology-based local fault detection in DC Microgrid clusters. Electric Power Systems Research. 2020 Dec 1:106981.

[2] H. R. Baghaee, M. Mirsalim, G. B. Gharehpetian, and H. A. Talebi, “A decentralized power management and sliding mode control strategy for hybrid AC/DC microgrids including renewable energy resources," IEEE Trans. Ind. Inform., pp. 1-1, March 2017.

[3] Kamel, Rashad Mohammedeen, Aymen Chaouachi, and Ken Nagasaka. "Comparison the performances of three earthing systems for micro-grid protection during the grid connected mode." Smart Grid and Renewable Energy, vol. 2, no. 3, 206, 2011.

[4] Mirsaeidi, Sohrab, et al. "Progress and problems in micro-grid protection schemes." Ren. and Sust. Energy Reviews, vol. 37, pp. 834-839, 2014.

[5] R. Mohanty and A. K. Pradhan, "DC Ring Bus Microgrid Protection Using the Oscillation Frequency and Transient Power," in IEEE Systems Journal, vol. 13, no. 1, pp. 875-884, March 2019.

[6] V. Nougain and B. K. Panigrahi, "Detection of DC System Faults Based on the Principle of Threshold Violation in i-r plane," in IEEE Systems Journal, doi: 10.1109/JSYST.2020.3010370.

[7] A. Meghwani, R. Gokaraju, S. C. Srivastava and S. Chakrabarti, "Local Measurements-Based Backup Protection for DC Microgrids Using Sequential Analyzing Technique," in IEEE Systems Journal, vol. 14, no. 1, pp. 1159-1170, March 2020.
[8] Y. Yang, C. Huang and Q. Xu, "A Fault Location Method Suitable for Low-Voltage DC Line," in IEEE Transactions on Power Delivery, vol. 35, no. 1, pp. 194-204, Feb. 2020.

[9] N. Bayati, H. R. Baghaee, A. Hajizadeh and M. Soltani, "Localized Protection of Radial DC Microgrids with High Penetration of Constant Power Loads," in IEEE Systems Journal, doi: 10.1109/JSYST.2020.2998059.

[10] L. Kong and H. Nian, "Fault Detection and Location Method for Meshtype DC Microgrid using Pearson Correlation Coefficient," in IEEE Transactions on Power Delivery, doi: 10.1109/TPWRD.2020.3008924.

[11] N. Bayati, A. Hajizadeh and M. Soltani, "Protection in DC microgrids: a comparative review," in IET Smart Grid, vol. 1, no. 3, pp. 66-75, 102018.

[12] N. Bayati, H. R. Baghaee, A. Hajizadeh and M. Soltani, "A Fuse Saving Scheme for DC Microgrids With High Penetration of Renewable Energy Resources," in IEEE Access, vol. 8, pp. 137407-137417, 2020.

[13] Park, J.D., Candelaria, J., Ma, L., et al.: 'DC ring-bus microgrid fault protection and identification of fault location', IEEE Trans. Power Deliv., vol. 28, no. 4, pp. 2574-2584, 2013.

[14] Christopher, E., Sumner, M., Thomas, D., et al. 'Fault location in a zonal DC marine power system using active impedance estimation', IEEE Trans. Appl. Ind., vol. 49, no. 2, pp. 860-865, 2013.

[15] Nanayakkara, O.M.K.K., Rajapakse, D.A., Wachal, R.: 'Travelingwavebased line fault location in star-connected multiterminal HVDC systems'. IEEE Trans. Power Deliv., vol. 27, no. 4, pp. 2286-2294, 2012.

[16] Fletcher, Steven DA,et al."High-speed differential protection for smart DC distribution systems." IEEE Transactions on Smart Grid, vol. 5, no. 5, pp. 2610-2617, 2014

[17] Feng, X., Qi, L., Pan, J.: 'A novel fault location method and algorithm for DC distribution protection', IEEE Trans. Ind. Appl., vol. 53, no. 3, pp. 1834-1840, 2017.

[18] S. D. A. Fletcher, P. J. Norman, S. J. Galloway, and G. M. Burt, "Determination of protection system requirements for DC unmannedaerial vehicle electrical power networks for enhanced capability and survivability," IET Elect. Syst. Transp., vol. 1, no. 4, pp. 137-147, Dec. 2011.

[19] D. Salomonsson, L. S* oder, and A. Sannino, "Protection of low-voltage dc microgrids," IEEE Trans. on Power Delivery, vol. 24, no. 3, pp. 10451053, 2009.

[20] R. Cuzner, D. MacFarlin, D. Clinger, M. Rumney, and G. Castles, "Circuit Breaker Protection Consideration in Power Converter-Fed DC Systems," IEEE Electric Ship Technologies Symposium, pp. 360-367, April 2009.

[21] S.D.A. Fletcher, P.J. Norman, S.J. Galloway, and G.M. Burt, "Determination of protection system requirements for DC unmanned aerial vehicle electrical power networks for enhanced capability and survivability," IET Electrical Systems in Transportation, vol. 1, no. 4, pp. 137-147, June 2011.

[22] D.R. Doan, "Arc Flash Calculations for Exposures to DC Systems," Industry Applications, IEEE Trans. on, vol. 46, no. 6, pp. 2299-2302, Nov./Dec. 2010

[23] Makkieh A, Psaras V, Peña-Alzola R, Tzelepis D, Emhemed A, Burt G. Fault location in DC microgrids based on a multiple capacitive earthing scheme. IEEE Journal of Emerging and Selected Topics in Power Electronics. 2020 May 20.

[24] S. Dhar, R. K. Patnaik and P. K. Dash, "Fault Detection and Location of Photovoltaic Based DC Microgrid Using Differential Protection Strategy," in IEEE Transactions on Smart Grid, vol. 9, no. 5, pp. 43034312, Sept. 2018.

[25] Abdali A, Mazlumi K, Noroozian R. High-speed fault detection and location in DC microgrids systems using Multi-Criterion System and neural network. Applied Soft Computing. 2019 Jun 1;79:341-53.

[26] Zhang L, Tai N, Huang W, Wang Y. Fault distance estimation-based protection scheme for DC microgrids. The Journal of Engineering. 2019 Apr 22;2019(16):1199-203.

[27] Mohanty, R., Balaji, U.S.M., Pradhan, A.K.: 'An accurate noniterative fault-location technique for low-voltage DC microgrid', IEEE Trans. Power Deliv.,2016, 31, (2), pp. 475-481.

[28] J. Yang, J. E. Fletcher, and J. O'Reilly, "Short-circuit and ground faultanalyses and location in VSC-based DC network cables,"IEEE Trans.Ind. Electron., vol. 59, no. 10, pp. 3827-3837, Oct. 2012. 